International Journal of Pure and Applied Mathematics

Volume 93 No. 5 2014, 685-698

ISSN: 1311-8080 (printed version); ISSN: 1314-3395 (on-line version)

url: http://www.ijpam.eu

doi: http://dx.doi.org/10.12732/ijpam.v93i5.8

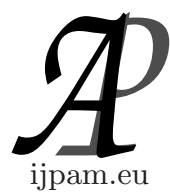

\title{
NEW GLOBAL CONVERGENCE OF NONMONOTONE LINE SEARCH ALGORITHM
}

\author{
Tahar Bouali $^{1} \S$, Yamina Laskri ${ }^{2}$, Rafik Guefaifia ${ }^{3}$ \\ $1,2,3$ Department of Mathematics \\ Badji Mokhtar University \\ Annaba, 23000, ALGERIA
}

\begin{abstract}
In this paper, a class of nonmonotone line search, study the convergence properties of such an algorithm for general nonconvex function, and proved its global convergence. More conjugate gradient algorithm is used the Wolfe rule nonmonotone line search. The global convergence results are proved.
\end{abstract}

AMS Subject Classification: 65K05, 90C30

Key Words: conjugate gradient, nonmonotone line search, global convergence, unconstrained optimization

\section{Introduction}

Our problem is to minimize a function of $n$ variables

$$
\min \left\{f(x): x \in \mathbb{R}^{n}\right\}
$$

where $f: \mathbb{R}^{n} \rightarrow \mathbb{R}$ is smooth and its gradient $g(x)$ is available. Conjugate gradient method for solving (1.1) are iterative methods of the form

$$
x_{k+1}=x_{k}+\alpha_{k} d_{k}
$$

where $\alpha_{k}>0$ is a steplength, $d_{k}$ is asearch direction. Normally the search direction at the first iteration is the steepest descent direction, namely $d_{1}=-g_{1}$.

Received: March 13, 2014

(C) 2014 Academic Publications, Ltd.

$\S_{\text {Correspondence author }}$ url: www.acadpubl.eu 
The other search direction can be defined recursively:

$$
d_{k}=-g_{k}+\beta_{k} d_{k-1}, k \geq 1
$$

where $g_{k}=\nabla f\left(x_{k}\right), \alpha_{k}$ is a stepe-size obtained by some line search, and $\beta_{k}$ is a scalar There are many ways to select $\beta_{k}$, and some well-known formulas are given by

$$
\begin{gathered}
\beta_{k}^{P R P}=\frac{g_{k}^{T} y_{k}}{\left\|g_{k-1}\right\|^{2}} \\
\beta_{k}^{F R}=\frac{\left\|g_{k}\right\|^{2}}{\left\|g_{k-1}\right\|^{2}} \\
\beta_{k}^{C D}=\frac{\left\|g_{k}\right\|^{2}}{-d_{k-1}^{T} g_{k-1}} \\
\beta_{k}^{D Y}=\frac{\left\|g_{k}\right\|^{2}}{d_{k-1}^{T} y_{k-1}} \\
\beta_{k}^{H S}=\frac{g_{k}^{T}\left(g_{k}-g_{k-1}\right)}{d_{k-1}^{T}\left(g_{k}-g_{k-1}\right)} \\
\beta_{k}^{N}=\frac{g_{k}^{T} y_{k-1}}{d_{k-1}^{T} g_{k-1}}-2 g_{k}^{T} d_{k-1} \frac{\left\|y_{k-1}\right\|^{2}}{\left(d_{k-1}^{T} g_{k-1}\right)^{2}}
\end{gathered}
$$

respectively, where $y_{k-1}=g_{k}-g_{k-1}$ and \|\| means the Euclidean norm. The technique of nonmonotone line search was proposed first in[1] and has received many successful applications or extensions in both unconstrained optimization and constrained optimization, A large portion of optimization methods require monotonicity of the objective values to guarantee their global convergence.This target is usually achieved by a suitable line technique even when the initial point is far away from the optimum. Among the most popular line search techniquesare are the Armijo rule, the Goldstein rule and the Wolfe rule (see[4, 5, 13]).In particular, enforcing monotonicity may considerably reduce the rate of convergence, when the iteration is trapped near a narrow curved valley, which can result in very short steps or zigzagging. Therefore, it might be advantageous to allow the iterative sequence to occasionally generate points with nonmonotone objective values, while retaining global convergence of the minimization algorithm.Several numerical tests show that the nonmonotone line search technique for unconstrained optimization and constrained optimization is efficient 
and competitive[ $[7,12,14]$. Note that the famous watchdog technique for constrained optimization proposed in [2] can also be viewed as strategy of the nonmonotone type. The forcing function introduced in [11] is an important class of functions which can be used to measure sufficiency of descent and prove convergence.In [11] a detailed steplength analysis with forcing function is given.Han and Liu [9] used the idea of forcing function and proposed a general line search rule. We combine forcing functions with the nonmonotone line search technique and give a general line search rule, called the nonmonotone F-rule, for unconstrained minimization problems. We show that some common nonmonotone line search rules such as the nonmonotone such as the nonmonotone Armijo line search rule, the nonmonotone Goldstein line search rule, and the Wolf line search rule are special cases of the nonmonotone F-rule. Finally, we prove the global convergence of the resulted nonmonotone descent methods under mild conditions. The remainder of this paper is organized as follows. In Section 2 we describe our nonmonotone F-rule and show that the aforementioned common nonmonotone line search rules, are particular cases of the nonmonotone F-rule.In Section 3, we establish the global convergence of nonmonotone descent methods for unconstrained optimization.Some conclusions are given in Section 4 .

In this paper, a class of non-monotone line search (NLS), study the convergence properties of such an algorithm for general nonconvex function, and proved its global convergence. This article studies the global convergence of a class of nonmonotone line search of Wolf rule conjugate gradient algorithm to prove that the idea comes from the on the F-rule search techniques. We study the condition of global convergence of four conjugate gradient methods with nonmonotone line searchs. When the conditions is being increased, for nonconvex functions, we prove the global convergence of modified method.

In the convergence analysis and implementation of conjugate gradient method, the extended wolf rule nonmonotone line search, namely

$$
\begin{gathered}
f\left(x_{k}+\alpha_{k} d_{k}\right) \leq \max _{0 \leq j \leq m(k)} f\left(x_{k-j}\right)+\gamma_{1} \alpha_{k} g_{k}^{T} d_{k} \\
\gamma_{2} g_{k}^{T} d_{k} \leq g\left(x_{k+} \alpha_{k} d_{k}\right)^{T} d_{k}
\end{gathered}
$$

which $0<\gamma_{1} \leq \gamma_{2} \leq 1, \lambda_{1}>0$ and $M \in N$,

$$
0 \leq m(k) \leq \min \{m(k-1)+1, M\}, m(0)=0
$$

Next, we present the nonmonotone F- rule. We begin with two definitions the forcing function and the reverse modulus of continuity of gradient.. 


\section{Technique of the Nonmonotone Line Search}

First given the general assumption of this section:

\section{Assumption 2.1.}

$\left(\mathrm{A}_{1}\right)$ The level set $L_{0}=\left\{x \backslash f(x) \leq f\left(x_{0}\right), x \in R^{n}\right\}$ is bounded, where $x_{0}$ is the starting point

$\left(\mathrm{A}_{2}\right) f$ is strongly convex and differentiable in the level set $L_{0}$ and its gradient $g_{k}=\nabla f\left(x_{k}\right)$ lipschitz continuous . ie, there exist constants $L>0$ making

$$
\|g(x)-g(y)\| \leq L\|x-y\|, \forall x, y \in \mathcal{N}
$$

Definition 2.2. The function $\sigma:[0, \infty) \rightarrow[0, \infty)$ is a forcing (F-function), if for any sequence $\left\{t_{i}\right\} \subset[0, \infty)$

$$
\lim _{k \rightarrow \infty} \sigma\left(t_{i}\right)=0 \text { implies } \lim _{k \rightarrow \infty} t_{i}=0
$$

Definition 2.3. Let

$$
\eta=\sup \left\{\|g(x)-g(y)\| / x, y \in L_{0}\right\}>0 .
$$

Then the mapping $\delta:[0, \infty) \rightarrow[0, \infty)$ defined by

$$
\delta(t)=\left\{\begin{array}{lr}
\inf \left\{\frac{\|x-y\|}{\|g(x)-g(y)\|} \geq t\right\}, & t \in[0, \eta) \\
\lim _{s \rightarrow \eta^{-}} \delta(s), & t \in[0, \eta)
\end{array}\right.
$$

is the reverse modulus of continuity of gradient $g(x)$.

Now we give the nonmonotone F-rule for line searches as follows.

$$
f\left(x_{k}+\alpha_{k} d_{k}\right) \leq \max _{0 \leq j \leq m(k)} f\left(x_{k-j}\right)-\sigma\left(t_{k}\right)
$$

where $\sigma$ is a forcing function and $t_{k}=-\frac{g_{k}^{T} d_{k}}{\left\|d_{k}\right\|}$. Set $x_{k+1}=x_{k}+\alpha_{k} d_{k}$

Obviously, if $M=0$, the above nonmonotone F-rule is just the rule of sufficient decrease in [11].

Note also that any nondecreasing function $\sigma:[0, \infty) \rightarrow[0, \infty)$ such that $\sigma(0)=0$ and $\sigma\left(t_{i}\right)>0$ for $t>0$ is necessarily an F-function.Hence, the presented rule is quite general.

For convenience, in the following, let:

$$
f\left(x_{k}+\alpha_{k} d_{k}\right) \leq \max _{0 \leq j \leq m(k)} f\left(x_{k-j}\right)
$$


where

$$
k-m(k) \leq l(k) \leq k
$$

Using Definition 2.3, we have

$$
\alpha_{k}\left\|d_{k}\right\| \geq \delta\left[\left(\gamma_{2}-1\right) \frac{g_{k}^{T} d_{k}}{\left\|d_{k}\right\|}\right]
$$

which means

$$
\alpha_{k}\left\|d_{k}\right\| \geq \delta\left[\left(1-\gamma_{2}\right)\left(-\frac{g_{k}^{T} d_{k}}{\left\|d_{k}\right\|}\right)\right]
$$

where $\delta($.$) is the reverse modulus. So, it follows from (1.4) and (2.4) that$

$$
\begin{gathered}
f\left(x_{k}+\alpha_{k} d_{k}\right) \leq f\left(x_{l(k)}\right)+\gamma_{1} \alpha_{k} g_{k}^{T} d_{k} \\
f\left(x_{k}+\alpha_{k} d_{k}\right)=f\left(x_{l(k)}\right)-\gamma_{1} \alpha_{k}\left\|d_{k}\right\|\left(-\frac{g_{k}^{T} d_{k}}{\left\|d_{k}\right\|}\right) \\
f\left(x_{k}+\alpha_{k} d_{k}\right) \leq f\left(x_{l(k)}\right)-\gamma_{1}\left(-\frac{g_{k}^{T} d_{k}}{\left\|d_{k}\right\|}\right) \delta\left[\left(1-\gamma_{2}\right)\left(-\frac{g_{k}^{T} d_{k}}{\left\|d_{k}\right\|}\right)\right] \\
f\left(x_{k}+\alpha_{k} d_{k}\right) \leq f\left(x_{l(k)}\right)-\sigma\left(-\frac{g_{k}^{T} d_{k}}{\left\|d_{k}\right\|}\right)
\end{gathered}
$$

where $\sigma(t)=\gamma_{1} t \delta\left[\left(1-\gamma_{2}\right) t\right], t \geq 0$. Clearly, $\sigma(t)$ is a forcing function. This indicates that the rule (1.4) - (1.5) satisfies the nonmonotone F-rule (2.3)

Algorithm 2.4. Given $\rho>0, \beta \in[0,1], \gamma_{2} \in[0,1], M$ non-negative integer, and to make the initial test step

$$
r_{k}=\frac{\rho g_{k}^{T} d_{k}}{\left\|d_{k}\right\|^{2}}
$$

Take $\alpha_{k}=\beta^{h(k)} r_{k}, h(k)=0,1,2, \ldots, h(k)$ is to make smallest non-negative integer set up by the following formula,

$$
f\left(x_{k}+\alpha_{k} d_{k}\right) \leq \max _{0 \leq j \leq m(k)} f\left(x_{k-j}\right)-\sigma\left(t_{k}\right)
$$

where $m(0)=0,0 \leq m(k) \leq \min \{m(k-1)+1, M\}, k \geq 1$. Obviously, if the descent direction $d_{k}$ is met $d_{k}^{T} g_{k}$, then, when sufficiently large $m(k)$, inequality (2.3) always, holds, thus satisfying $\alpha_{k}$ the condition of existence.

The above line search in each iteration, it is recommended that the initial test step $r_{k}$ no longer remain the same, but can be adjusted automatically. On 
the value, change the initial test approach can get better results, calculte a larger step

size $\alpha_{k}$, there by reducing the number of iterations.

\section{Remarks 2.5}

1- In order to be able to use a nonmonotonic line search the NLS calculate the step length facor $\alpha_{k}$ must be the search direction $d_{k}$ is a descent direction. In the next

section, we will prove that this study of the conjugate gradient algorithm to keep the search direction $d_{k}$ is down.

2- In order to able to calculate a larger step size $\alpha_{k}$.

$$
\sum_{k=0}^{\infty} \sigma\left(t_{k}\right)<\infty
$$

\section{Convergence Analysis}

In this section we establish the global convergence properties of optimization methods with nonmonotone F-rule.Note that to establish our result, we need some additional mild conditions.

Lemma 3.1. Let the search direction $d_{k}$ is a descent direction and step size factor $\alpha_{k}$ by the nonmonotone line search NLS by (1.2) of iteration $\left\{x_{k}\right\} \subset L_{0}$.

Proof. By the nonmonotone line search in the NLS (2.3) show

$$
\begin{gathered}
f\left(x_{1}\right) \leq f\left(x_{0}\right)-\sigma\left(t_{0}\right)<f\left(x_{0}\right) \\
f\left(x_{2}\right) \leq \max _{0 \leq j \leq m(1)} f\left(x_{1-j}\right)-\sigma\left(t_{1}\right)<f\left(x_{0}\right) \\
f\left(x_{3}\right) \leq \max _{0 \leq j \leq m(2)} f\left(x_{2-j}\right)-\sigma\left(t_{2}\right)<f\left(x_{0}\right) \\
\ldots \ldots \\
f\left(x_{k+1}\right) \leq \max _{0 \leq j \leq m(k)} f\left(x_{k-j}\right)-\sigma\left(t_{k}\right)<f\left(x_{0}\right)
\end{gathered}
$$

As a result $\left\{x_{k}\right\} \subset L_{0}$ 
Lemma $3.2[8]$. The step factor $\alpha_{k}$ NLS1 of nonmonotone line search, by (1.2), amendment to the definition of method to meet

$$
g_{k}^{T} d_{k} \leq-\frac{7}{8}\left\|g_{k}\right\|^{2}, k=0,1,2 \ldots
$$

Proof. Since $d_{0}=-g_{0}$, we have $g_{0}^{T} d_{0}=-\left\|g_{0}\right\|^{2}$

$$
g_{k}^{T} d_{k}=-\left\|g_{k}\right\|^{2}+\beta_{k} g_{k}^{T} d_{K-1}
$$

when $\beta_{k}=\beta_{k}^{N}$

$$
\begin{gathered}
g_{k}^{T} d_{k}=-\left\|g_{k}\right\|^{2}+\beta_{k}^{N} g_{k}^{T} d_{k-1} \\
-g_{k}^{T} d_{k}=\left\|g_{k}\right\|^{2}-g_{k}^{T} d_{k-1}\left(\frac{g_{K}^{T} y_{k-1}}{-g_{k-1}^{T} d_{k-1}}-2 g_{k}^{T} d_{k-1} \frac{\left\|y_{k-1}\right\|^{2}}{\left(-g_{k-1}^{T} d_{k-1}\right)^{2}}\right) \\
=\frac{\left\|g_{k}\right\|^{2}\left(-g_{k-1}^{T} d_{k-1}\right)^{2}-g_{k}^{T} d_{k-1} g_{k}^{T} y_{k-1}\left(-g_{k-1}^{T} d_{k-1}\right)+2\left(g_{k}^{T} d_{k-1}\right)^{2}\left\|y_{k-1}\right\|^{2}}{\left(-g_{k-1}^{T} d_{k-1}\right)^{2}}
\end{gathered}
$$

we apply the inequality

$$
u^{T} v \leq \frac{1}{2}\left(\|u\|^{2}+\|v\|^{2}\right)
$$

to the second term in (3.2) with

$$
u=\frac{1}{2} g_{k}\left(-g_{k-1}^{T} d_{k-1}\right), v=2\left(g_{k}^{T} d_{k-1}\right) y_{k-1}
$$

Therefore, we can get that (3.1) is true for all $k \in N$.

For simplicity, we introduce the notation

$$
l(k)=\max \left\{i \mid 0 \leq k-i \leq m(k), f\left(x_{i}\right)=\max _{0 \leq j \leq m\langle k)} f\left(x_{k}-j\right)\right\}
$$

Namely $l(k)$ is non-negative integer, and satisfy the following two formulas

$$
\begin{gathered}
k-m(k) \leq l(k) \leq k \\
f\left(x_{l(k)}\right) \underset{0 \leq j \leq m(k)}{=\max _{0}} f\left(x_{k-j}\right)
\end{gathered}
$$


So nonmonotone line search NLS1 (1.4) can be rewritten as

$$
f\left(x_{k}+\alpha_{k} d_{k}\right) \leq f\left(x_{l(k)}\right)-\sigma\left(t_{k}\right)
$$

Lemma 3.3. Under the conditions of the assumptions $\left(A_{1}\right)$, the sequence $f\left(x_{l(k)}\right)$ is decreases monotonically.

Proof. By (2.3), knowledge of all $k$

$$
f\left(x_{k+1}\right) \leq f\left(x_{l(k)}\right)
$$

have been established. Nonmonotine line search NLS, $0 \leq m(k) \leq m(k-1)+$ 1,therefore:

$$
\begin{gathered}
f\left(x_{l(k)}\right)=\max _{0 \leq j \leq m(k)} f\left(x_{k-j}\right) \leq \max _{0 \leq j \leq m(k-1)+1} f\left(x_{k-j}\right) \\
=\max \left\{\max _{0 \leq j \leq m(k-1)} f\left(x_{k-1-j}\right), f\left(x_{k}\right)\right\} \\
=\max \left\{f\left(x_{l(k-1)}\right), f\left(x_{k}\right)\right\} \\
=f\left(x_{l(k-1)}\right)
\end{gathered}
$$

Lemma 3.4. Assuming $\left(\mathrm{A}_{1}\right)$ holds, then the $f\left(x_{l(k)}\right)$ converges i.e $\lim _{k \rightarrow \infty} f\left(x_{l(k)}\right)$ exists.

and

$$
\lim _{k \rightarrow \infty} \sigma\left(t_{l(k)-1}\right)=0
$$

Proof. Known $f(x)$ is bounded below . Since $f\left(x_{k}\right) \leq f\left(x_{0}\right), \forall k,\left\{x_{k}\right\} \subset$ $L_{0}$ (see proo Lemma 3.1 ) and $\left\{f\left(x_{l(k)}\right)\right\}$ decreases monotonically (see proo Lemma 3.3 ), then

$\lim _{k \rightarrow \infty} f\left(x_{l(k)}\right)$ exists. By (3.6)

$$
f\left(x_{l(k)}\right) \leq f\left(x_{l(l(k)-1}\right)-\sigma\left(t_{l(k)-1}\right)
$$

On both sides of order $k \rightarrow \infty$ that it. So

$$
\lim _{k \rightarrow \infty} \sigma\left(t_{l(k)-1}\right)=0
$$

Theorem 3.5. Let function $f: \mathbb{R}^{n} \rightarrow \mathbb{R}$ satisfy Assumption 2.1. Let the sequence $\left\{x_{k}\right\}$ be defined by (1.2)

where the steplength $\alpha_{k}$ is defined by the nonmonotone F-rule (2.3). If the direction $d_{k}$ satisfies 


$$
-\frac{g_{k}^{T} d_{k}}{\left\|d_{k}\right\|} \geq \sigma\left(\left\|g_{k}\right\|\right), k=0,1, \ldots,
$$

and

$$
\left\|d_{k}\right\| \leq m_{1}\left\|g_{k}\right\|
$$

where $\sigma(\cdot)$ is a forcing function and $m_{1}>0$. Then the sequence $\left\{x_{k}\right\} \subset L_{0}$ and

$$
\lim _{k \rightarrow \infty}\left\|g_{k}\right\|=0
$$

Proof. Which means from Definition 2.2 that

$$
\lim _{k \rightarrow \infty} t_{l(k)-1}=-\frac{g_{l(k)-1}^{T} d_{l(k)-1}}{\left\|d_{l(k)-1}\right\|}=0
$$

Using condition (3.8), we deduce

$$
\lim _{k \rightarrow \infty} \sigma\left(\left\|g_{l(k)-1}\right\|\right)=0
$$

which implies

$$
\lim _{k \rightarrow \infty}\left\|g_{l(k)-1}\right\|=0
$$

from Definition 2.2. Then it follows from (3.9) that

$$
\lim _{k \rightarrow \infty}\left\|d_{l(k)-1}\right\|=0
$$

Let

$$
l_{1}(k)=l(k+M+2)
$$

We prove by induction that for any given $j \geq 1$

$$
\lim _{k \rightarrow \infty}\left\|d_{l_{1}(k)-j}\right\|=0
$$

and

$$
\lim _{k \rightarrow \infty} f\left(x_{l_{1}(k)-j}\right)=\lim _{k \rightarrow \infty} f\left(x_{l(k)}\right)
$$


If $j=1$, since $\left\{l_{1}(k)\right\} \subset\{l(k)\},(3.13)$ and (3.14) follow from (3.11). Assume that (3.13) and (3.14) hold for a given $j$. We consider the case of $j+1$. Since

$$
\left.f\left(x_{l_{1}(k)-j}\right) \leq f\left(x_{l\left(l_{1}(k)-(j+1)\right.}\right)\right)-\sigma\left(\gamma_{l_{1}(k)-(j+1)}\right)
$$

using the same argument for deriving (3.11), we deduce

$$
\left.\lim _{k \rightarrow \infty} \| d_{l_{1}(k)-(j+1)}\right) \|=0
$$

Know (3.13) was established. But

$$
x_{l_{1}(k)-j}-x_{l_{1}(k)-(j+1)}=\alpha_{l_{1}(k)-(j+1)} d_{l_{1}(k)-(j+1)}
$$

Noting that $L_{0}=\left\{x \backslash f(x) \leq f\left(x_{0}\right), x \in R^{n}\right\}$ is bounded, $x_{k+1}=x_{k}+\alpha_{k} d_{k}$ $\in L_{0}$,for all $k$ and that $\alpha_{k}$ stay bounded, we have

$$
\lim _{k \rightarrow \infty}\left\|x_{l_{1}(k)-j}-x_{l_{1}(k)-(j+1)}\right\|=0
$$

$f(x)$ was uniformly continuous on the level $L_{0}$, which

$$
\begin{gathered}
\lim _{k \rightarrow \infty} f\left(x_{l_{1}(k)-(j+1)}\right)=\lim _{k \rightarrow \infty} f\left(x_{l_{1}(k)-j}\right) \\
=\lim _{k \rightarrow \infty} f\left(x_{l_{1}(k)}\right)=\lim _{k \rightarrow \infty} f\left(x_{l(k)}\right)
\end{gathered}
$$

This shows that the arbitrary $j \geq 1$. (3.16) have also set up By $L_{1}$ definition and (3.4) are available

$$
L_{1}(k)=L(k+M+2) \leq k+M+2
$$

namely

$$
L_{1}(k)-k-1 \leq M+1
$$

Thus, for any $k$, do deformed

$$
\begin{aligned}
x_{k+1} & =x_{L_{1}(k)}-\sum_{j=1}^{L(k)-k-1}\left(x_{l_{1}(k)-j+1}-x_{l_{1}(k)-j}\right) \\
& =x_{L_{1}(k)}-\sum_{j=1}^{L(k)-k-1} \alpha_{l_{1}(k)-j} d_{l_{1}(k)-j}
\end{aligned}
$$


where $x_{L_{1}(k)}$ transposition, and noting (3.17), was

$$
\left\|x_{k+1}-x_{L_{1}(k)}\right\|=\left\|-\sum \lim i t s_{j=1}^{L(k)-k-1} \alpha_{l_{1}(k)-i} d_{l_{1}(k)-i}\right\| \leq \sum_{j=1}^{M+1}\left\|\alpha_{l_{1}(k)-i} d_{l_{1}(k)-i}\right\|
$$

On both sides of order $k \rightarrow \infty$, by (3.17),

$$
\lim _{k \rightarrow \infty}\left\|x_{k+1}-x_{L_{1}(k)}\right\|=0
$$

Thus, by the uniform continuity of $f(x), \lim _{k \rightarrow \infty} f\left(x_{l_{1}(k)}\right)=\lim _{k \rightarrow \infty} f(x)$, then from (3.19), we can see

$$
\lim _{k \rightarrow \infty} f\left(x_{l(k)}\right)=\lim _{k \rightarrow \infty} f\left(x_{K}\right)
$$

So, for

$$
f\left(x_{k+1}\right) \leq f\left(x_{l(k)}\right)-\sigma\left(\frac{-g_{k}^{T} d_{k}}{\left\|d_{k}\right\|}\right)
$$

taking limits for $k \rightarrow \infty$, we get

$$
\lim _{k \rightarrow \infty} \sigma\left(-\frac{g_{k}^{T} d_{k}}{\left\|d_{k}\right\|}\right)=0
$$

which means

$$
\lim _{k \rightarrow \infty}\left\|g_{k}\right\|=0
$$

Corollary 3.6. Let function $f: \mathbb{R}^{n} \rightarrow \mathbb{R}$ satisfy Assumption 2.1. Let the sequence $\left\{x_{k}\right\}$ be defined by (1.2) where the steplength $\alpha_{k}$ is defined by the nonmonotone F-rule

(2.3) with $F$-function $\sigma(t)=\left(\frac{m_{2}}{m_{1}}\right) t$, and the direction $d_{k}$ satisfies

$$
g_{k}^{T} d_{k} \leq m_{2}\left\|g_{k}\right\|^{2}
$$

and

$$
\left\|d_{k}\right\| \leq m_{1}\left\|g_{k}\right\|
$$

where $m_{1}, m_{2}>0$. Then the sequence $\left\{x_{k}\right\} \subset L_{0}$ and 


$$
\lim _{k \rightarrow \infty}\left\|g_{k}\right\|=0
$$

Proof. ( This follows directly from [3] and Theorem 3.5)

Corollary 3.7. Under the conditions of the assumptions 2.1. Consider any iterative method (1.2), where $d_{k}$ satisfies $(3.23)-(3.24)$ and $\alpha_{k}$ is obtained by the nonmonotone line search $(1.4)-(1.5)$

Then, there exists a constant $m_{3}$ such that

$$
\left\|g_{k+1}\right\| \leq m_{3}\left\|g_{k}\right\|, \text { for all } k
$$

Further, we have that

$$
\lim _{k \rightarrow \infty}\left\|g_{k}\right\|=0
$$

Proof. Noting that $\alpha_{k} \leq \lambda_{1}$, by this, (1.2), and (3.24) we have that

$$
\begin{gathered}
\left\|x_{k+1}-x_{K}\right\|=\left\|\alpha_{k} d_{k}\right\| \leq \alpha_{k}\left\|d_{k}\right\| \\
\leq \lambda_{1} m_{1}\left\|g_{k}\right\|
\end{gathered}
$$

and from the lipschitz continuity (2.1) and (3.27), we can get that

$$
\begin{gathered}
\left\|g_{k+1}-g_{K}\right\| \leq L\left\|x_{k+1}-x_{k}\right\| \leq L \lambda_{1} m_{1}\left\|g_{k}\right\| \\
\left\|g_{k+1}\right\| \leq\left(L \lambda_{1} m_{1}+1\right)\left\|g_{k}\right\|
\end{gathered}
$$

Thus, (3.25) holds with

$$
m_{3}=L \lambda_{1} m_{1}+1
$$

In addition, it follows by $(2.6),(3.23),(3.24)$ and $(3.25)$, that (3.26) holds 


\section{Conclusions}

The program prepared by the Matlab6.5 in general on a PC. Test functions from [10], indicated in brackets after the function name is the number of variables. NLS is a line search method proposed in this paper, by GLL on Grippo- Lampariello-Lucidi from non-monotonic line search. $n_{i}$ represents the number of iterations, $n_{f}$ the number of times that the function value, gradient calculation is the number $n_{i}+1$. We were calculated for different values of $M$, when $M=0$, ie, monotone line search.To the pros and cons of the algorithm, the parameters are uniform taken as, $\sigma=1, \beta=0.2, \gamma_{2}=0.9, \varepsilon=10^{-6}$.Our conjugate gradient method is divided into two kinds of numerical experiment for a class of initial testing step according to this formula to the case of correction, the other is the case of an initial test step length fixed for a. From the results of the comparison, the proposed line search termination criterion has the following advantages:

1 -Monotone line search $(M=0)$, or non-monotone line search $(M>0)$, the number of iterations of the NLS method, the function value calculation times are reduced.

2-Usually better than the initial test step fixed the case when the initial testing step according to this formula be amended.

3-Non-monotone strategy is effective for most of the functions, especially high-dimensional, or initial testing step fixed the situation

\section{References}

[1] Al-Baali, M. Descent property and global convergence of the FletcherReeves method with inexact line search. IMA J. Numer. Anal. 5 (1985), no. 1, 121-124. dx.doi.org/10.1093/imanum/5.1.121.

[2] R.M. Chamberlain, C. Lemarechal, H.C. Pedersen, M.J.D. Powell. The watchdog technique for forcing convergence in algorithms for constrained optimization, Math Progrmming Stude. 16(1982) 1-17. dx.doi.org/10.1007/BFb0120945.dx.doi.org/10.1007/BFb0120945

[3] Y. H. Dai. On the Nonmonotone Line Search, Journal of Optimization theory and Applications: Vol. 112, No. 2, pp. 315-330, February 2002

[4] J.E. Dennis, R.B. Schnabel. Numerical Methods for Unconstrained Op- 
timization and Nonlinear Equations, Prentice-Hall, Englewood Clijs, NJ, 1993.

[5] R.Fletcher. Practical Methods of Optimization, 2nd Edition, Wiley, New York, 1987.

[6] L.Grippo., F.Lampariello, S.Lucidi. A Nonmonotone line search technique for Newton's method, SIAM Journal on Numerical Analysis, Vol. 23, pp. 707-716 (1986). dx.doi.org/10.1137/0723046

[7] L.Grippo, F.Lampariello, S.Lucidi. A truncated Newton method with nonmonotone line search for unconstrained, optimization, J.Optim.Theory Appl.60 (1989) 401-419. dx.doi.org/10.1007/BF00940345

[8] W.W.Hager, H.Zhang. A new conjugate gradient method with guaranteed descent and an efficient line search. SIAM J Optim. 2005, 16:170-192

[9] J.Han, L.Liu. General form of stepsize selection rules of line search and relevant analysis of global convergence of BFGS algorithm, Acta Math.Appl.Sinica 18 (1995)112-12

[10] J.J.Moré, B.S.Garbow, K.E.Hillstrom. Testing unconstrained optimization software, ACM Transactions on mathematical software., 17-41, (1981). ACM0098-3500/81/0300-0017

[11] J.M. Ortega, W.C. Rheinboldt. Iterative Solution of Nonlinear Equations in Several Variables, Academic Press, NewYork, 1970.

[12] E.R. Panier, A.L. Tits. Avoid the Maratos eject by means of nonmonotone line search, I, general constrained problems, SIAM J.Numer.Anal.28 (1991) 1183-1195.

[13] W.Sun, R.J.B.Sampaio, J.Yuan. On trust region algorithm for nonsmooth optimization, Appl.Math.Comput.85 (1997) 109-116.

[14] P.L.Toint. An assessment of nonmonotone line search techniques for unconstrained optimization, SIAM J.Sci. Comput.17 (1996) 725-739. 